\title{
„Das Ganze durch eine geheime und gewissermaßen unbekannte Kette zu verbinden." Zivilisierung und polyperspektivischer Briefroman bei Montesquieu und Wieland
}

"De lier de tout par une chaîne

secrette \&, en quelque façon, inconnue ». Civilisation et multiplication des perspectives dans le roman épistolaire de Montesquieu et Wieland

"To connect the whole by a secret and, as it were, undiscoverable chain". Civilisation and the polyperspectival epistolary novel

Jutta Heinz

\section{(2) OpenEdition} Journals

Édition électronique

URL : http://journals.openedition.org/ceg/1021

DOI : $10.4000 /$ ceg. 1021

ISSN : 2605-8359

Éditeur

Presses Universitaires de Provence

Édition imprimée

Date de publication : 30 mai 2016

Pagination : 251-266

ISBN : 979-10-320-0067-0

ISSN : 0751-4239

Référence électronique

Jutta Heinz, " „Das Ganze durch eine geheime und gewissermaßen unbekannte Kette zu verbinden.”

Zivilisierung und polyperspektivischer Briefroman bei Montesquieu und Wieland », Cahiers d'Études Germaniques [Online], 70 | 2016, Online erschienen am: 17 Dezember 2017, abgerufen am 25 November 2020. URL : http://journals.openedition.org/ceg/1021; DOI : https://doi.org/10.4000/ceg. 1021 


\title{
„Das Ganze durch eine geheime und gewissermaßen unbekannte Kette zu verbinden.“
}

\author{
Zivilisierung und polyperspektivischer \\ Briefroman bei Montesquieu und Wieland
}

Jutta HEINZ

Wieland-Forschungszentrum Oßmannstedt

Auf den ersten Blick scheinen der französische Schriftsteller, Philosoph und Staatstheoretiker Charles-Louis de Secondat, Baron de La Brède et de Montesquieu, und der deutsche Schriftsteller, Dichter und Essayist Christoph Martin Wieland wenig miteinander zu tun zu haben. Montesquieu wird 1689 in eine Familie des hohen Amtsadels in Südfrankreich geboren; seine Lebenszeit umfasst die erste Hälfte des 18. Jahrhunderts, er stirbt in dem Jahr, in dem Jean-Jacques Rousseau seinen epochemachenden ersten Discours sur l'origine et les fondements de l'inégalité parmi les hommes (1755) veröffentlicht. Christoph Martin Wieland wird knapp fünfzig Jahre später, im Jahr 1733, in der süddeutschen Freien Reichsstadt Biberach in eine bürgerliche Familie von Pastoren und Ratsherren geboren; seine Lebenszeit erstreckt sich über die zweite Hälfte des 18. Jahrhunderts, und er stirbt im Jahr der Völkerschlacht von Leipzig (1813). Auf den zweiten Blick entdeckt man jedoch einige nicht unwesentliche Gemeinsamkeiten. Beide veröffentlichten sowohl philosophische als literarische Werke, wobei Montesquieu wohl eher als dichtender Philosoph und Wieland als philosophierender Dichter zu beschreiben wäre; aber letztlich sind diese Grenzen für beide sowieso notwendig künstlich und unscharf. ${ }^{1}$ Beide interessieren sich in hohem Maße für Politik, besonders für die Fragen nach der besten Regierungsform und nach der kulturellen Entwicklung der Menschheit. Und beide weisen in ihrem

1. Die Charakterisierung, die Rudolf Vierhaus von Montesquieus Person und Werk vornimmt, könnte auch für Wieland stehen: „Montesquieu war ein Mann von bedeutender Gelehrsamkeit, die gleichwohl die Regeln des Geschmacks und die Anforderungen der Eleganz des Ausdrucks gewichtiger nahm als das Gebot der Exaktheit und Vollständigkeit - ein Mann von ,Welt', in der das Politische wie bei den Alten im Mittelpunkt stand.“ Rudolf Vierhaus, „Montesquieu in Deutschland. Zur Geschichte seiner Wirkung als politischer Schriftsteller im 18. Jahrhundert“, in ders., Deutschland im 18. Jahrhundert. Politische Verfassung, soziales Gefüge, geistige Bewegungen, Göttingen, Vandenhoeck \&t Ruprecht, 1987, S. 9-32, hier S. 10 f.; dort auch umfassend zur Wirkungsgeschichte und den Übersetzungen von Montesquieus Werken. 
poetischen Euvre ein auch im an Briefroman reichen 18. Jahrhundert nicht eben häufig vertretenes Genre auf, nämlich den polyperspektivischen Briefroman. ${ }^{2}$

Auch im Blick auf die Briefromane drängen sich zunächst Unterschiede auf. Montesquieus Lettres persanes, die 1721 anonym erschienen und bald verboten wurden, machten dessen ungeachtet den jungen Autor berühmt und lösten eine Welle an Nachahmungen aus. Wielands Aristipp und einige seiner Zeitgenossen, ein Alterswerk, publiziert in vier Bänden achtzig Jahre später (1800-1802), fanden ein eher schwaches öffentliches Echo. Wichtiger sind aber die Gemeinsamkeiten: Beide Texte sind „Reisebriefwechselromane“ ${ }^{3}$, die die Erfahrungen von nach Alter, Geschlecht und kultureller Herkunft unterschiedlichen Figuren auf ihren Erkundungsreisen schildern. Sie bedienen sich dazu umfangreicher Quellen aus Ethnologie und Geschichte und präsentieren dem Leser eine Fülle von Wissensbeständen und gelehrten Anspielungen; und sie behandeln eine Reihe für die Aufklärung grundlegender philosophischer und kulturtheoretischer Themen.

Wie jedoch hängt die spezifische literarische Form des polyperspektivischen Briefromans mit diesen spezifischen Fragestellungen zusammen? Dieser Frage werde ich im Folgenden nachgehen, indem ich die Lettres persanes und den Aristipp-Roman in dreierlei Hinsicht vergleiche. Als erstes geht es um die Erzählstruktur sowie das jeweilige poetologischeKonzept des polyperspektivischen Briefromans. In einem zweiten Schritt werde ich auf die Troglodyten-Episode in den Persischen Briefen genauer eingehen, die Wieland 1791 übersetzt hat; sie kann als narrativ konzentrierte Darstellung der Positionen beider Autoren zur Entwicklungsgeschichte der Menschheit verstanden werden. Zum Dritten werde ich die Auseinandersetzung mit Geschlechterfragen in beiden Romanen daraufhin untersuchen, wie in ihr Privates und Öffentliches zusammenhängen und wie sie mit den kulturtheoretischen Fragestellungen verbunden ist. Am Ende sollte daraus ersichtlich werden, welche Funktionen der polyperspektivische Briefroman als Genre für den Zivilisationsprozess erfüllt.

\section{"Die Freiheit, philosophische, politische und moralische Gedanken in einen Roman einzufügen" - Poetologie und Struktur des polyperspektivischen Briefromans}

Montesquieus Lettres persanes umfassen in der ersten Ausgabe von 1721150 und in der 1758 posthum von Montesquieus Sohn herausgegebenen Ausgabe

2. Vgl. zum Problem der Zuspitzung des Gattungskonstrukts ,Briefroman' in der Forschung auf den empfindsamen Briefroman Gideon Stiening, Epistolare Subjektivität. Das Erzählsystem in Friedrich Hölderlins Briefroman ,Hyperion oder der Eremit in Griechenland, Tübingen, Niemeyer, 2005; auch Stiening benennt Montesquieu als einen der wichtigsten und frühesten Zeugen eines explizit nicht empfindsamen Briefromans (vgl. S. 27).

3. Vgl. die umfassende Darstellung bei Hans-Peter Nowitzki, „Anthropologie und Gattungspoetik“, in Gideon Stiening/Robert Vellusig (Hrsg.), Poetik des Briefromans. Wissens- und mediengeschichtliche Studien, Berlin / New York, de Gruyter, 2012, S. 219-260. 
161 Briefe. $^{4}$ Insgesamt 19 Briefschreiber richten ihre Briefe an insgesamt 24 Empfänger; ${ }^{5}$ den größten Teil tragen der ältere Reisende Usbek mit insgesamt 79 Briefen und sein jüngerer Landsmann Rica mit 45 bei. Zehn Briefe werden von Frauen und Eunuchen aus dem Harem verfasst. Rund 60 der Briefe sind dem Orient gewidmet, ihnen stehen 100 gegenüber, die sich mit den Erfahrungen der Figuren im Okzident befassen. Aus der inneren Chronologie kann man schließen, dass die Reise im Jahr 1711 in Isfahan in Persien beginnt und mit der Revolte im dortigen Harem wahrscheinlich im Jahr 1720 endet. Die Briefe werden aus Venedig, Russland, Spanien, England, Schweden und Frankreich verschickt; all diese Länder, ihre Kulturen, ihre Sitten, ihre Religionen und politischen Verfassungen, werden zum Gegenstand kulturvergleichender Überlegungen der Briefschreiber. Drei philosophische Erzählungen sind eingeschaltet, deren ersten die Troglodyten-Geschichte ist. ${ }^{6}$ Einige Briefsequenzen bilden thematisch zusammenhängende Komplexe wie beispielsweise die Briefe über Bevölkerungsentwicklung oder die Satire auf die Finanzexperimente von John Law in England.

Schon diese reine Beschreibung macht deutlich, dass es sich bei dem Text wahrlich um einen „enzyklopädischen Roman“ ${ }^{7}$ handelt, der den zeitgenössischen Leser auf eine tour de force durch die real- und geistesgeschichtliche Welt des frühen 18. Jahrhunderts schickt. Dabei wird er aus der fiktiven Perspektive des fremden, orientalischen Reisenden mit einer Reihe von unliebsamen Wahrheiten über die eigene westliche Welt konfrontiert. Denn obwohl Usbek als aufgeklärter Orientale nach Westen fährt, da er nicht der Meinung ist, dass „nur das Licht des Orients uns erleuchten dürfe“ “8 findet er auch unter der hellen Sonne der Aufklärung noch reichlich obskure gesellschaftliche, religiöse und politische Gebräuche. Die erste, strategische Funktion des polyperspektivischen Briefromans ist es, die Kritik dieser Gebräuche und Institutionen, vor allem in heiklen Religionsfragen, dadurch zu entschärfen, dass der Herausgeber ja nur als

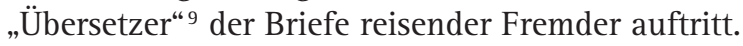

Auf die zweite Funktion des Genres weist Montesquieu in einer später hinzugefügten Bemerkung zu den Persischen Briefen (1754) hin. Der polyperspektivische Briefroman mit seinem breiten Figurenpanorama bietet ihm nicht nur eine Vielfalt von interkulturellen Perspektiven auf die Welt dar, hinter denen der Autor verschwindet und zwischen denen der Leser sich selbst situieren

4. Der Roman wird hier zitiert nach der deutschen Übersetzung von Peter Schunck, Stuttgart, Reclam, 2004 (im Folgenden zitiert mit der Sigle PS); zur komplizierten Editionsgeschichte und den verschiedenen Fassungen vgl. das dortige Nachwort, S. 305; zur Forschungsliteratur die Literaturhinweise (S. 353f.). Die französischen Zitate sind den EEuvres Complètes de Montesquieu entnommen (Bd. 1, Oxford, Voltaire Foundation, 2004; im Folgenden zitiert mit der Sigle LP).

5. Vgl. zur quantitativen Auswertung Schunck, Nachwort, S. 360 f.

6. Siehe unten II.

7. So Birgit Wagner, "Strategien der Wissensselektion in den Lettres persanes“, in Stiening/ Vellusig, Briefroman, S. 35-48, hier: S. 35; dort auch ausführlich zu den Quellen sowie zur Forschungsliteratur.

8. PS, S. 13; „que la lumiere Orientale dût seule nous éclairer“ (LP, S. 141).

9. PS, S. 9; LP, S. 139: „traducteur“. 
muss; er ermöglicht es erst strukturell, genuin philosophische Reflexionen im Roman zu vermitteln. Montesquieu führt aus:

Letztlich sind in gewöhnlichen Romanen nur dann Abschweifungen möglich, wenn sie selbst wieder einen Roman bilden. Gedanken und Überlegungen haben keinen Platz im gewöhnlichen Roman; dies würde dem Plan und der Art des Werks zuwiderlaufen, da die Personen nicht dafür gemacht sind, Überlegungen anzustellen. Dagegen handelt es sich im Briefroman nicht um frei gewählte Personen, und die Gegenstände folgen keinem vorgefertigten Plan; der Verfasser hat daher die Freiheit, philosophische, politische und moralische Gedanken in einen Roman einzufügen und das Ganze durch eine geheime und gewissermaßen unbekannte Kette zu verbinden. ${ }^{10}$

Die Ketten-Metapher ${ }^{11}$ (eine Variante des alten Topos der great chain of being oder scala naturae) ist zentral: Sie unterstellt einen engen kausalen, aber auf der Oberfläche unsichtbaren Zusammenhang, der nicht nur die Handlung, sondern auch Philosophie, Politik und Moral untereinander und mit dieser zu einem „Ganzen“ verbindet. Im Gegensatz zur philosophischen Abhandlung gibt es dabei keinen ausgezirkelten „Plan“, keine strenge logische Abfolge der Argumente. Der Verfasser bewahrt sich vielmehr seine „Freiheit“ als Autor und gewinnt dadurch die Möglichkeit, den Leser auch bei abstrakten Themen emotional zu involvieren, indem er politische Theorien in konkreten Staatszusammenhängen, moralische Überzeugungen samt ihrer Wirkung im Alltag und philosophische Systeme in ihren Grenzen und Möglichkeiten zeigt.

Wielands Altersroman Aristipp und einige seiner Zeitgenossen umfasst etwas weniger Briefe als die Persischen Briefe, nämlich 144; zudem dominiert der Namensgeber Aristipp mit 82 verfassten und 52 empfangenen Briefen. Nach ihm kommt schon eine Frau, nämlich die weibliche Hauptfigur Lais mit je 26 geschriebenen und empfangenen Briefen. Ansonsten präsentieren sich auch hier die Briefschreiber bunt gemischt nach Alter, Geschlecht und Herkunft; es handelt sich teilweise um reale historische Personen wie Sokrates und Aristipp, teils um fiktive Figuren. Die Titelfigur durchreist die antike Welt zwischen seinem Geburtsort Kyrene, Ägina, Milet, Korinth, Syrakus, Samos, Rhodos und Athen als Zentrum; mit den jeweiligen Orten sind auch verschiedenen Stadtkulturen und politische Verfassungen verbunden. Der Zeitraum umfasst ungefähr die

10. PS, S. 5; „Enfın, dans les romans ordinaires, les digressions ne peuvent être permises que lorqu'elles forment elles-même un nouveau roman. On n’y sçauroit mêler de raisonnemens, parce qu'aucuns des personnages n'y avant été assemblés pour raisonner, cela choqueroit le dessein Et la nature de l'ouvrage. Mais, dans la forme de lettres, où les acteurs ne sont pas choisis, \& où les sujets qu'on traite ne sont dependans d'aucun dessein ou d'aucun plan déjà formé, l'auteur s'est donné l'avantage de pouvoir joindre de la philosophie, de la politique \&t de la morale, à un roman ; $C t$ de lier le tout par une chaîne secrette $\&$, en quelque façon, inconnue“ (LP, S. 567 f.).

11. Einige Zeilen vorher heißt es bereits: „Die einzelnen Personen sind miteinander verbunden wie mit einer Kette“ (ibid.; „des divers personnages sont placés dans une chaîne qui les lie“, LP, S. 567); vgl. zur erkenntnistheoretischen Funktion dieser Figur auch: Montesquieu, Vom Geist der Gesetze, hrsg. und übersetzt von Kurt Weigand, Stuttgart, Reclam, 2006, S. 92: „Viele Wahrheiten werden erst einsichtig, wenn die Gedankenkette sichtbar wird“). 
Jahre zwischen 404 und 435 v. Chr. Wieland benutzt eine Vielzahl antiker Quellen, die teilweise in Anmerkungen erläutert werden. Die Stelle des Fremden, die bei Montesquieu der Orient einnahm, ist hier also die dem gebildeten zeitgenössischen Leser nicht ganz so fremde Welt der griechischen Antike in ihrem goldenen Zeitalter. Innerhalb dieses etwas reduzierten geographischen Kosmos ist jedoch die gleiche,enzyklopädische' Breite der Themen und Bezüge gegeben wie bei Montesquieu: Diskutiert werden eine Vielzahl philosophischer Themen, Kunst und Literatur, Religion und Sitten, soziale Beziehungen im Allgemeinen und Geschlechterfragen im Besonderen, und vor allem Politik; allein der Auseinandersetzung mit Platons politeia ist beinahe das gesamte vierte Buch des Romans gewidmet. ${ }^{12}$

Zwar schreibt Wieland zum Aristipp kein Vorwort, aber im Roman wird eine Reihe poetologischer Debatten geführt, die sich unschwer auf den Text selbst beziehen lassen. So vergleicht Aristipp gleich anfangs seine Briefe an seinen Freund Kleonidas mit einer im 18. Jahrhundert geläufigen Metapher mit einem „Spaziergang“:

Du wirst dich, wie ich sehe, schon daran gewöhnen müssen, lieber Kleonidas, daß ich nicht lange in meinem Wege fortgehen kann, ohne bald auf diesen bald auf jenen Gegenstand zu stoßen, der mich zu einer kleinern oder größern Abschweifung verleitet. In sofern ich dir nur keine Langeweile mache, wird es dir übrigens gleichviel sein, was für einen Weg ich dich führe, da meine Briefe bloße Spaziergänge für dich sind. ${ }^{13}$

Wieland rechtfertigt damit ebenso wie Montesquieu in seiner Bemerkung zu den persischen Briefen die im Text enthaltenen Abschweifungen; und er nimmt sich die gleiche Freiheit als Autor, nicht logisch-stringent, sondern frei assoziierend mit seinen Themen zu verfahren. Dabei integriert er, ebenfalls wie Montesquieu, verschiedene literarische Genres: Die Briefe können Dialoge enthalten, kleine Geschichten, größere zusammenhängende Abhandlungen wie die politeia-Kritik. Eines der wichtigsten formalen Vorbilder für ihn ist die antike Symposienliteratur. Im Aristipp-Roman finden mehrere Symposien statt; Lais, die sie ausrichtet, erläutert den besonderen Charakter dieser halb öffentlichen, halb privaten Veranstaltungen:

Mein Haus ist, zwar nicht zu allen Stunden, aber doch in den gewöhnlichen, wo man Gesellschaft sieht, allen offen, die man zu Athen Kalokagathen nennt. Eupatriden, Staatsund Kriegsmänner, Dichter, Sofisten und Künstler, alte und junge, reiche und arme, fremde und einheimische, jedermann, der sich in guter Gesellschaft mit Anstand zeigen kann, ist gern gesehen. ${ }^{14}$

12. Vgl. insgesamt zur Struktur und den Hauptthemen des Aristipp Jutta Heinz, Narrative Kulturkonzepte. Wielands ,Aristipp' und Goethes ,Wilhelm Meisters Wanderjahre' (Heidelberg, Winter, 2006), in der die hier nur skizzenhaft ausgeführten Analysen zum Aristipp-Roman ausführlicher nachgelesen werden können.

13. Christoph Martin Wieland, Aristipp und einige seiner Zeitgenossen, hrsg. von Klaus Manger, Frankfurt a.M., Hanser, 1988, S. 44 (im Folgenden zitiert mit der Sigle AZ).

14. AZ, S. 384. 
Die Symposien zehren also sehr von der gemischten Gesellschaft, die sich dort trifft, wobei die wesentliche Eintrittsbedingung ist, dass die Teilnehmer „Kalokagathen“ sind: schöne und freie Geister, die sich unter angenehmen äußeren Umständen in eine „freie muntre Unterhaltung über interessante Gegenstände“ 15 einlassen. Diese darf jedoch keinesfalls pedantisch und akademisch werden, sondern muss auch bei schwergewichtigen philosophischen Themen immer die „Freiheit und Willkürlichkeit eines Symposischen Gesprächs“" ${ }^{16}$ wahren:

\begin{abstract}
Ausführliche methodische Behandlung und Erschöpfung des Stoffes der Unterredung schickt sich auf keine Weise für ein Gespräch dieser Art; aber desto lobenswürdiger ist es, wenn die redenden Personen, indem sie nur mit leichtem Fuß über den Gegenstand hin zu glitschen scheinen, dennoch alles sagen, was den Zuhörer auf den Grund der Sache blicken läßt, und in den Stand setzt, sich jede Frage, die noch zu tun sein könnte, selbst zu beantworten. ${ }^{17}$
\end{abstract}

Die Formulierung vom „Zuhörer“ legt schon nahe, dass damit auch der Leser eines solchen symposischen Gesprächs - das letztlich nur die kleine Variante des großen symposischen Gesprächs ist, das der Roman selbst darstellt - direkt angesprochen wird. Will er am symposischen Gespräch jedoch vollwertig teilnehmen, muss er sich ebenso wie die Teilnehmer im Roman an den Kommunikations- und Verhaltensidealen orientieren, die den gesamten Aristipp-Roman durchziehen: der Urbanität, verstanden als gleichzeitig ethisch und moralisch ausgebildete Lebensform des Stadt- wie des Weltbürgers ${ }^{18}$ ebenso wie der eng mit ihr verbundenen Mäßigung, die für Wieland (wie für Montesquieu) eine Quintessenz antiker Lebensweisheit darstellt. ${ }^{19}$

\title{
Die Geschichte der Troglodyten - „Bei manchen Wahrheiten genügt die Überzeugung nicht“
}

Wie jedoch entsteht die interkulturell vielfältige und urban gemäßigte Kommunikation, die Montesquieus und Wielands Briefromane idealtypisch zeichnen, die selbstja wiederum ein Zeugnis nicht nur akademischer Gelehrsamkeit, sondern Ausdruck hoher Bildung und philosophischer Reflektiertheit ihrer Autoren sind? Tatsächlich ist die Entstehung von Kultur im Sinne dessen, was in der Zeit auch als,Poliziertheit' oder civilité bezeichnet wird ${ }^{20}$, ein wichtiges Thema in beiden Romanen. Eng verbunden ist sie mit der philosophischen Frage nach dem,Naturzustand' des fiktiven Urmenschen: Ist der Mensch von Natur aus gut? Hat er von Natur aus gesellige Instinkte, ist er empathiefähig? Hat er gar ein natürliches Gefühl für Gut und Böse, ein,moralisches Gefühl'? Von der Antwort auf diese Fragen hängt die gesamte Konzeption der weiteren Entwicklung der

15. AZ, S. 385.

16. AZ, S. 553.

17. AZ, S. 568.

18. Vgl. Heinz, Narrative Kulturkonzepte, Kap. 5.4.2.

19. Vgl. Heinz, Narrative Kulturkonzepte, Kap. 5.5.3.

20. Vgl. zu den Konzepten von „Kultur’ und ,Zivilisation’ Heinz, Narrative Kulturkonzepte, Kap. 2.3. 
Menschheit, sowohl politisch als auch anthropologisch, ab. Und wie Mitte des Jahrhunderts für Rousseau ist sie bereits für Montesquieu - mangels konkreter Überlieferungen - nur durch ein Gedankenexperiment zu beantworten: Dazu dient die im 11. bis 14. Brief der Persischen Briefe erzählte Geschichte der Troglodyten als eines fiktiven Naturvolks. ${ }^{21}$ Der Erzählung geht dabei die theoretische Frage voraus, für die die Geschichte selbst dann das Exempel liefert; Mirza berichtet an Usbek: „Gestern wurde die Frage aufgeworfen, ob die Menschen glücklich sind aufgrund des Vergnügens und der Befriedigung der Sinne oder aufgrund tugendhaften Verhaltens“. ${ }^{22}$ Statt nun die seit der Antike bekannten philosophischen Antworten auf diese Frage auszukramen, erzählt Usbek lieber eine Geschichte und begründet dies auch - nämlich mit einer für das Verhältnis von Moral, Philosophie und Literatur zentralen Überlegung:

bei manchen Wahrheiten genügt die Überzeugung nicht, man muß auch erreichen, daß sie empfunden werden. Zu diesen Wahrheiten gehören die der Moral. Vielleicht spricht Dich die folgende kurze Geschichte mehr an als philosophische Überlegungen. ${ }^{23}$

Die Entwicklung der Troglodyten, die Usbek im Folgenden berichtet, geht dabei nicht ganz von einem Naturzustand aus. Vielmehr werden gleich zu Beginn zwei Regierungsformen erprobt - die Monarchie und die Republik -, die jedoch sofort an der „eigenen wilden Natur“ 24 der noch vollständig unzivilisierten Troglodyten gewaltsam scheitern. Daraus ziehen diese den Schluss, dass fortan jeder nur noch seine eigenen Interessen verfolgen soll - es entsteht also eine Art Hobbesscher Krieg von allen gegen alle. Anstelle nun die fatalen Folgen dieser Entscheidung theoretisch zu analysieren, werden fünf Beispiele aufgereiht, die die fatalen Auswirkungen dieses gesetz- und rechtlosen Zustands ganz konkret aufzeigen: Es geht um die Ungleichheit der natürlichen Ressourcen (I), die Verteilung der Frauen (II), den mangelnden Schutz des Eigentums (III), um Probleme beim Tauschhandel (IV) und um das Fehlen eines Gesundheitssystems (V). Am Ende präsentiert der auswärtige Arzt, der die egoistischen Troglodyten zunächst vor einer schweren Epidemie gerettet hatte, nach Nicht-Bezahlung seines Honorars aber eine erneute Behandlung verweigerte, seinen Patienten die eigentliche Rechnung mitsamt der Diagnose:

21. Die Bezeichnung erscheint erstmals bei Herodot, der einen Stamm in Süden Libyens, der sich angeblich von Reptilien ernährt, Troglodytai Aithiopes nennt. Weitere Historiker und frühe Ethnographen griffen die Bezeichnung auf, dabei wurden die Geschichten von den Troglodyten immer mehr mythologisch angereichert.

22. PS, S. 27 ("Hier on mit en question, si les hommes étoient heureux par les plaisirs, \& les satisfactions de Sens ; ou par la pratique de la vertu ?", LP, S. 159).

23. PS, S. 28 ("il y a de certaines verités qu'il ne suffit pas de persuader, mais qu'il faut encore faire sentir; telles sont les verités de Morale ; peut-être que ce morceau d'histoire te touchera plus qu'une Philosophie subtile“, LP, S. 161).

24. Ibid. ("son naturel sauvage“, LP, S. 162). 
In eurer Seele ist ein tödlicher wirkendes Gift als das, von dem ihr Heilung sucht! Ihr verdient keinen Platz auf der Erde, weil ihr keine Menschlichkeit besitzt und die Regeln dessen, was recht und billig ist, nicht kennt. ${ }^{25}$

Die Troglodyten können nur durch einen kompletten Neuanfang nach dem biblischen Muster der Sintflut gerettet werden: Die Seuche rafft auf wundersame Weise alle sittlich verderbten Troglodyten hinweg und lässt nur zwei Familien übrig, die ganz un-troglodytisch sind: „Sie besaßen Menschlichkeit, kannten Gerechtigkeit, liebten die Tugend ${ }^{\text {“ }}{ }^{26}$ Zudem siedeln sie, ein klassisches UtopieMotiv, im „abgelegensten Winkel des Landes“27 und haben gute Böden. Ihr gesamtes Leben wird nach dem Muster der Idylle als eine Art goldenes Zeitalter dargestellt: Die Natur selbst beschenkt sie mit ihren Früchten, zwischen den Ehepartnern herrscht die zärtlichste Liebe, die Kinder wachsen wohlerzogen auf, es werden ländliche Feste zu Ehren der Götter veranstaltet und die „Freuden des Landlebens und das Glück eines Lebens frei von Schuld“ ${ }^{28}$ bewahren sie vor den Versuchungen von Reichtum und Luxus. Besonders wichtig für diese Idylle ist aber genau das, was die anderen Troglodyten in ihr Verderben gestürzt hatte: ihre außergewöhnliche Fähigkeit zur Empathie, zum gegenseitigen Wohlwollen. Alle Troglodyten betrachten sich nämlich, wiederum mit einem klassischen Topos formuliert, als „eine einzige Familie“.29 Und aus diesem natürlichen Gemeinschaftsgefühl heraus entwickeln sie in einer ersten Reflexion auch die entscheidende Erziehungsmaxime für ihren Nachwuchs, die im diametralen Gegensatz zur früheren Troglodyten-Maxime des vollständigen Egoismus steht:

\footnotetext{
Sie machten ihnen vor allem verständlich, daß das Interesse der einzelnen im Interesse der Gemeinschaft liegt, daß es den eigenen Untergang bedeutet, wenn man sich von ihr trennen möchte. ${ }^{30}$
}

Das goldene Zeitalter kann aber bekanntlich nicht ewig dauern, und so erwachsen auch den Troglodyten zwei Gefahren, von denen sie die erste noch souverän meistern: Sie werden von außen bedroht durch ein barbarisches Nachbarvolk, aber ihre Liebe zur Gemeinschaft ebenso wie ihr Familiensinn begeistern sie zu ungeahnten Kampfeskräften. Die wahre Gefahr jedoch droht von innen. Das Volk wächst nämlich zu stark, man beschließt eines Tages, dass man doch einen Anführer braucht, und so wollen die Troglodyten einvernehmlich den Gerechtesten, einen in der Tugend bewährten Greis, zu einer Art Philosophenkönig machen. Dieser jedoch lehnt den Antrag geradezu empört ab: Denn offensichtlich sei er ein

25. PS, S. 31 (“vous avez dans l'ame un poison plus mortel, que celui dont vous voulez guerier ; vous ne meritez pas d'occuper une place sur la terre; parce que vous n'avez point d'humanité, \&t que les regles de l'équité vous sont inconnues“, LP, S. 164).

26. Ibid. ("ils avoient de l'humanité ; ils connaissoient la justice ; ils aimoient la vertu“, LP, S. 165).

27. PS, S. 32 (“dans l'endroit du païs le plus écarté“, LP, S. 165).

28. PS, S. 33 ("les delices de la vie champêtre, \& le bonheur d'une condition toujours parée de l'innocence“, LP, S. 167).

29. PS, S. 34 ("comme une seule famille“, LP, S. 168).

30. PS, S. 32 ("ils leur faisoient sur tout sentir que l'interêt des particuliers se trouve toujours dans l’interêt commun ; que vouloir s'en separer, c'est vouloir se perdre !“, LP, S. 166). 
Zeichen dafür, dass seinen geliebten Troglodyten ihre „Tugendhaftigkeit“ eine „Last“" werde -

In der Lage, in der ihr seid, das heißt ohne Anführer, müßt ihr wider Willen tugendhaft sein. Sonst könntet ihr nicht bestehen und würdet in die elende Lage eurer Vorväter zurückfallen. Aber dieses Joch erscheint euch zu drückend; ihr wäret lieber einem Fürsten unterworfen und würdet seinen Gesetzen gehorchen, die weniger streng sind als eure Sitten. ${ }^{31}$

Das Argument ist frappierend und zeugt von dialektischer Schulung: Wirklich frei, so zeigt der Greis seinen Mitbürgern, sind sie nur, solange jeder sich selbst regiert. Sobald sie die Verantwortung für ihre eigene Sittlichkeit aber an einen wie auch immer hervorragend qualifizierten Vertreter delegieren, machen sie sich selbst nicht nur unfrei, sondern entledigen sich ihrer grundlegenden moralischen Verantwortung als Menschen: Sie gehorchen nur noch mechanisch vorgegebenen Gesetzen, nicht mehr ihrem eigenen moralischen Urteil; ihre moralische Urteilskraft würde in dem Maße schrumpfen, wie sie nicht mehr gebraucht wird, und damit würde auch ihre Grundlage, das natürliche moralische Gefühl, überflüssig. Wer die freiwillig anerkannte Herrschaft der Tugend durch autoritätsgestützte Gesetze ersetzt, so befürchtet der Greis, der verliert seine moralische Freiheit am Ende ganz.

Allerdings haben sich sowohl die orientalische als auch die okzidentalische Welt im Roman weit von diesen Urformen menschlicher Vergesellschaftung entfernt; ein troglodytisches Dasein im besten Sinne kann kein politischer Entwurf für eine eher über-zivilisierte Gesellschaft im Osten wie im Westen oder für politische Großmächte sein. Was jedoch in dieser Ursprungs-Parabel gezeigt werden kann, sind die subtilen Mechanismen der Entstehung von Ungleichheit und Unfreiheit. Und was mit ihr bewirkt werden kann, ist eine auch emotionale Auseinandersetzung des Lesers mit den alltagsweltlichen, geradezu archetypisch geschilderten Troglodyten-Schicksalen: Es geht gleichzeitig um Erkenntnis und um Empathie - eben deshalb erzählt Usbek eine Geschichte und hält keinen gelehrten kulturhistorischen Vortrag.

Im Jahr 1790 übersetzt Wieland die Troglodyten-Geschichte und rückt sie in die Januar-Ausgabe des Neuen Teutschen Merkur ein ${ }^{32}$ es ist die Zeit seiner intensiven Auseinandersetzung mit der Französischen Revolution. Zunächst betont Wieland jedoch in seiner kurzen Einführung ebenfalls den parabolischen Charakter des Textes: Er habe versucht, aus den „Nachrichten meiner Vorgänger“ ${ }^{33}$

31. PS, S. 36 f. (“dans l'état où vous êtes, n'ayant point de Chef, il faut que vous soyez vertueux malgré vous ; sans cela vous ne sçauriez subsister, \&t vous tomberiez dans la malheur de vos premiers Peres : mais ce joug vous paroît trop dur, vous aimez mieux être soumis à un Prince, \&t obéïr á ses Loix moins rigides que vos mœurs“, LP, S. 171).

32. Neuer Teutscher Merkur 1790, 1, S. 33-47 (im Folgenden zitiert mit der Sigle NTM).

33. Genannt werden Montesquieu und der Engländer George Lyttelton (vgl. NTM, S. 33). Ein Vergleich mit Lytteltons Letters from a Persian in England to his Friend at Ispahan (1735), der die Erzählung von dem Zeitpunkt an, als Montesquieu abbricht, fortsetzt und mit vielen zeitgenössischen Bezügen auf England bereichert, würde weitere interessante interkulturelle Perspektiven ergeben, kann hier aber aus Platzgründen nicht stattfinden. 
und meinen eignen ein Ganzes zu machen [...], welches für die Liebhaber der moralischpolitischen Naturgeschichte des Menschen wenigstens in so fern einiges Interesse haben kann, als man daraus begreifen wird, wie es zugehen könne, daß ein Volk, nachdem es, von der rohesten Wildheit an, alle Stufen von Kultur durchlaufen hat, sich zulezt unvermerkt und zum großen Erstaunen der Zuschauer wieder auf dem nemlichen Punkte befinden könne, von welchem es ausgegangen ist. ${ }^{34}$

In vielen Details bleibt Wieland dabei sehr eng an Montesquieu und übersetzt beinahe wörtlich; es finden sich jedoch einige sehr interessante Ergänzungen und Abweichungen. Neu ist der explizite Bezug auf Rousseau, gleich zu Beginn. ${ }^{35}$ Zudem scheinen die Erfahrungen der Französischen Revolution überdeutlich dort durch, wo geschildert wird, wie sich die Troglodyten zu Beginn jeder Herrschaft, auch der republikanischen, entledigen: „Freiheit“ und „Gleichheit“ stehen im Zentrum der Passage, die im Ausruf „Es lebe die Freiheit!“ 36 gipfelt - wobei der kommentierende Erzähler gleich darauf hinweist, es handele sich allerdings nur um eine „unächte Freyheit“" ${ }^{37}$, die allein dazu diene, sich des „lästigen Jochs der bürgerlichen Verfassung“" 38 zu entledigen.

Besonders ausführlich beschreibt Wieland den Neuanfang durch die beiden auserwählten Familien, denen hier zusätzlich noch die Attribute der „Mäßigung und Arbeitsamkeit“39 zugeschrieben werden - zentrale Begriffe nicht nur für Wielands Lebensphilosophie, sondern auch ein Hinweis auf die Notwendigkeit konkreter Tätigkeit für die kulturelle Entwicklung. Zudem betont er die vorreflexive Basis der Moralität noch stärker als Montesquieu: Die guten Troglodyten sind „instinctmäßig und sympathetisch“ wohlwollend, gütig und gemeinschaftsbezogen. Sobald sie aber mit dem Nachdenken beginnen, „überzeugte“ sie auch „ihre Vernunft“

von der Richtigkeit ihres Gefühls, in dem sie einen jeden in den übrigen ein vervielfältigtes Ich erkennen lehrte, und es zu einer Sache, welche der Ausrechnung fähig war, machte, daß unsere eigene Glückseligkeit um so viel größer und vollkommner wird, je inniger unsre Theilnehmung an andern ist. ${ }^{40}$

Die Verbindung zwischen Eigeninteresse und Gemeinschaftsinteresse wird hier noch enger geknüpft als bei Montesquieu: Sie entspringt nicht nur einem natürlichen Instinkt, sondern kann gleichzeitig äußerst rational begründet werden, als Kalkül nämlich, das der „Ausrechnung“ fähig ist; die gleiche mathematische Metaphorik liegt auch der originellen Metapher vom „vervielfältigten Ich“ zugrunde. Und auch bei Wieland steht an entscheidender Stelle die Formel vom Volk der Troglodyten als „einziger Familie“. ${ }^{41}$ Schließlich

\author{
34. NTM, S. 34. \\ 35. Vgl. ibid. \\ 36. NTM, S. 36. \\ 37. NTM, S. 37. \\ 38. NTM, S. 36. \\ 39. NTM, S. 42. \\ 40. NTM, S. 43. \\ 41. NMT, S. 44. Die Formulierung ist bei Wieland schon in seinen frühesten politischen Schriften \\ nachweisbar und zieht sich durch sein Werk hindurch. Vgl. zur differenzierteren späteren \\ Auseinandersetzung mit dem Paternalismus Montesquieus Position im Geist der Gesetze, S. 103.
}


wird die Beschreibung der moralischen Erziehung der Kinder noch erweitert: Haben diese bei Montesquieu vor allem an den guten Vorbildern der Eltern gelernt, betont Wieland darüber hinaus den Wert abschreckender Beispiele sowie von Gewöhnung und Training: Die „mechanisch gewordene Fertigkeit“ zur Mäßigung der Leidenschaften ist die zweite „Grundlage der Tugend““ ${ }^{42}$

Leider bricht die Darstellung nach der zweiten Exempelkette ab; eine Fortsetzung wird zwar angekündigt, erscheint aber niemals. Insgesamt betrachtet sind sich Montesquieu und Wieland in den Grundzügen darüber einig, dass das Verständnis der Menschheit als einer einzigen Familie - das ja auch unter einer biologisch-evolutionären Perspektive sinnvoll ist - zumindest als moralisches Postulat nicht nur instruktiv, sondern letztlich anthropologisch am angemessensten ist: Wer nicht in der Lage ist, sein eigenes „Ich“ so zu „vervielfältigen“, dass er im Anderen sein eigenes verfremdetes Abbild sieht und in der politischen Gemeinschaft die Summe der Interessen ihrer Mitglieder, der ist entweder noch nicht reif für politische Organisationsformen oder bereits in seinen natürlichen moralischen Gefühlen korrumpiert.

\section{„Insere Herrschaft über die Frauen ist eine wahre Tyrannei" - der Harem als Modell der Despotie}

Wie eng privates und öffentliches Interesse, Moral und Politik, für beide Autoren nicht nur in der Familien-Metapher zusammenhängen, zeigt die besondere Bedeutung des Geschlechterdiskurses in den Persischen Briefen wie im Aristipp-Roman. Bei Montesquieu ist er zentral für die Haremshandlung, die die stärker theoretischen, kulturvergleichenden Passagen der Reise rahmt. ${ }^{43}$ In der Forschung ist bereits mehrfach darauf hingewiesen worden, dass der Harem ein verkleinertes Modell der Despotie ist: Eine Vielzahl verschiedener Frauen wird von einem männlichen Tyrannen beherrscht, der seine Schreckensherrschaft durch willfährige Diener, die Eunuchen, ausführen lässt. ${ }^{44}$ Aber es ist durchaus nicht so, dass der Despot dabei glücklich ist; Usbek erläutert vielmehr:

Es ist nicht so, Nessir, daß ich sie liebte: In dieser Hinsicht fühle ich so wenig, daß kein Verlangen aufkommt. In dem Serail, wo ich mit vielen Frauen gelebt habe, habe ich mich vor der Liebe geschützt und diese durch sich selbst zerstört. Aber gerade aus meiner Gefühlskälte entspringt eine geheime Eifersucht, die mich verzehrt. ${ }^{45}$

42. NTM, S. 44. Hingegen werden die Darstellung der Religion sowie der Idyllencharakter deutlich zurückgedrängt.

43. Vgl. dazu Claudia Opitz-Belakhal, Aufklärung der Geschlechter, Revolution der Geschlechterordnung. Studien zur Politik- und Kulturgeschichte des 18. Jahrhunderts, Münster, Waxmann, 2002, bes. Kap. 3.

44. Vgl. beispielsweise Henning Ottmann, Geschichte des politischen Denkens. Bd. 3: Neuzeit, Stuttgart/ Weimar, Metzler, S. 434.

45. PS, S. 18f. (“Ce n'est pas, Nessir, que je les aime : je me trouve à cet egard dans une insensibilité, qui ne me laisse point de desirs. Dans le nombreux Serrail, où j’ai vêcu, j’ai prévenu l'amour, $\mathrm{Ct}$ l'ai détruit par lui-même: mais de ma froideur même il sort une jalousie secrette, qui me devore“, LP, S. 149). 
Hingegen beklagen sich die Frauen nicht nur über die Grausamkeit der Männer im Allgemeinen und des Despoten insbesondere, sondern erkennen sogar die fatale Dialektik jeglicher Gewaltherrschaft. Sie weisen nämlich mehrfach darauf hin, dass sie der „Fühllosigkeit“46 des Despoten nicht vollständig ausgeliefert sind; so schreibt Zelis an Usbek:

Du darfst Dir aber nicht einbilden, Usbek, daß Deine Lage glücklicher sei als meine; denn ich habe tausend Vergnügungen genossen, die Du nicht kennst. Meine Phantasie war unaufhörlich damit beschäftigt, mir ihren Wert deutlich zu machen. Ich habe gelebt, aber Du hast nur Sehnsüchte empfunden. Selbst in dem Gefängnis, in dem Du mich festhältst, bin ich freier als Du. ${ }^{47}$

Selbst Usbek erkennt durch seine ja durchaus redliche Auseinandersetzung mit den andersartigen Geschlechterverhältnissen im Okzident zumindest theoretisch, dass die krasse Ungleichheit der Partner und die (äußerliche) Unfreiheit der einen Seite keine Basis für eine befriedigende Beziehung sind; so verteidigt er nämlich interessanterweise die Institution der Scheidung, weil ihr Verbot eine "freie Handlung, bei der das Herz einen großen Anteil haben sollte“, durch „Zwang, Notwendigkeit und das Verhängnis des Schicksals“48 zerstöre. Als jedoch der Harem durch seine lange Abwesenheit außer Kontrolle gerät und auch die den Eunuchen erteilten Generalvollmachten die Ordnung nicht mehr sicherstellen können, sieht Usbek endlich ganz konkret, was ihm bei einer Rückkehr drohen würde: Er selbst würde „sich in Mauern einschließen“ müssen, „die für mich noch schrecklicher sind als für die Frauen, die dort bewacht werden". ${ }^{49}$ Und ausgerechnet seine vermeintlich moralisch so gefestigte und treue Lieblingsfrau Roxane weist Usbek vor ihrem Selbstmord, der dem Roman ein trostloses Ende setzt, genau wie Zelis darauf hin, dass er sich selbst mit seiner Despotie der Freiheit beraubt habe:

Wie konntest Du nur denken, ich sei so einfältig und würde mir vorstellen, ich sei nur auf der Erde, um Deine Launen zu bewundern? Und Du hättest das Recht, alle meine Begierden zu unterdrücken, während Du selbst Dir alles erlaubst? Nein! Ich habe in der Knechtschaft leben können, aber ich war immer frei. Ich habe Deine Gesetze nach den Gesetzen der Natur abgeändert, und mein Geist hat sich immer seine Unabhängigkeit bewahrt. ${ }^{50}$

46. PS, S. 118 ("insensibilité“, LP, S. 296).

47. Ibid. (“Cependant, Usbek, ne t'imagine pas que ta situation soit plus heureuse que la mienne : j'ai gouté ici mille plaisirs, que tu ne connois pas: mon imagination a travaillé sans cesse à m'en faire connoître le prix : j’ai vécu, \&t tu n'as fait que languir. Dans la prison même, où tu me retiens, je suis plus libre que toi“, LP, S. 296).

48. PS, S. 214 ("Dans une action si libre, \&t où le cœur doit avoir tant de part, on mit la gêne, la necessité, \&t la fatalité du destin même“, LP, S. 444).

49. PS, S. 295 (“J'irai m'enfermer dans des murs plus terribles pour moi, que pour les femmes qui y sont gardées“, LP, S. 541).

50. PS, S. 300 (“Comment as-tu pensé que je fusse assez credule, pour m'imaginer que je ne fusse dans le monde, que pour adorer tes caprices? Que pendant que tu te permets tout, tu eusses le droit d'affliger tous mes desirs? Non : j'ai pû vivre dans la servitude, mais j'ai toujours été libre, j'ai reformé tes Loix sur celles de la nature; \&t mon esprit s'est toujours tenu dans l'independance“, LP, S. 544). 
Die Haremshandlung demonstriert damit nicht nur die offensichtliche menschenunwürdige Brutalität des Despotismus, sondern auch die verdeckte korrumpierende Wirkung absoluter Macht auf die Herrschenden selbst. Ein theoretisches Vorbild für gerechtere Geschlechterverhältnisse wären zwar die Troglodyten, aber in einer viel stärker zivilisierten Gesellschaft verliert es seine Gültigkeit. Aus westlicher Perspektive belehrt immerhin ein „sehr galanter Philosoph“ Rica in Paris, die Frauen seien sogar das bessere Geschlecht, da sie „mehr Menschlichkeit und Vernunft besitzen“. Die fatale gesellschaftliche Ungleichheit ist deshalb ihm zufolge nur durch das Generalheilmittel der Aufklärung schlechthin, die Erziehung nämlich, zu bekämpfen: „wenn die Erziehung die gleiche wäre, wären auch die Kräfte gleich“. ${ }^{51}$

Was jedoch wäre, wenn die Positionen von Männern und Frauen, Herrschern und Beherrschten, einmal ganz einfach vertauscht wären, demonstriert die im letzten Teil eingeschaltete persische Erzählung von Anais im weiblichen Paradies, wo „auch die tugendhaften Frauen an einen Ort der Freuden gelangen, wo sie sich mit göttlichen Männern, die ihnen untertan sind, in einem Strom wollüstiger Genüsse berauschen werden“. ${ }^{52}$ Wie schon in der Troglodyten-Geschichte greift Montesquieu hier auf das Mittel erzählender exemplarischer Veranschaulichung zurück, das eine stärkere emotionale Beteiligung der Leser sicherstellt - und darüber hinaus im Motiv des Rollentauschs einen Perspektivwechsel anbietet, der die Grundlage für eines zeitgemäßeres wechselseitiges Verständnis der Geschlechter bieten könnte.

Wielands Analyse des Geschlechterverhältnisses in Milieu der Antike entspricht ziemlich genau dem von Montesquieu im orientalischen Milieu; und die große emanzipierte Heldin des Aristipp-Romans, die ehemalige Hetäre Lais, spricht es unverblümt aus:

\footnotetext{
daß der weibliche Teil der Menschheit sich beinahe auf dem ganzen Erdboden in einem Zustande von Abwürdigung und Unterdrückung befindet, der sich auf nichts in der Welt als Überlegenheit der Männer an körperlicher Stärke gründen kann; da die Vorzüge des Geistes, in deren ausschließlichen Besitz sie sich zu setzen suchen, nicht ein natürliches Vorrecht ihres Geschlechts, sondern eine der Usurpazionen sind, deren sie sich kraft ihrer stärkeren Knochen über uns angemaßt haben. ${ }^{53}$
}

Doch Lais ist nicht die einzige starke Frau im Aristipp-Roman; ihr an die Seite gestellt werden eine Reihe von Geschlechtergenossinnen, die andere Bilder des Weiblichen verkörpern und andere Beziehungsmodelle erproben - Timandra als ideale Partnerin für eine ,offene Ehe', Musarion als perfekte Hausfrau und Künstlermuse, Kleone als klassische ,schöne Seele' und Seelenverwandte Aristipps. In den Töchtern von Kleone und Aristipp wird angedeutet, dass für die Zukunft auch eine Vereinigung von Emanzipation und eigenständigem

51. PS, S. 75 ("les forces seroient égales, si l'education l'etoit aussi“, LP, S. 232).

52. PS, S. 262 ("aussi les femmes vertueuses iront dans un lieu de delices, oú elles seront enyvrées d'un torrent de voluptez avec des hommes divins, qui leur seront soumis“, LP, S. 505).

53. AZ, S. 134. 
weiblichen Selbstbewusstsein möglich sein könnte. ${ }^{54}$ Die Schlüsselrolle dafür kommt nämlich auch bei Wieland der Erziehung zu; Lais beklagt den schlechten Ist-Zustand:

Du weißt vermutlich, wie wenig bei der Erziehung der Griechischen Töchter in Betrachtung kommt, daß sie auch eine Seele haben, und daß die Seele kein Geschlecht hat. Sie werden erzogen um so bald als möglich Ehfrauen zu werden; und der Grieche verlangt von seiner ehlichen Bettgenossin nicht mehr Geist, Talente und Kenntnisse, als sie nötig hat, um (wo möglich) schöne Kinder zu gebären, ihre Mägde in der Zucht zu halten, und die Geschäfte des Spinnrockens und Webstuhls zu besorgen. ${ }^{55}$

Daneben propagiert der Roman ein lebensweltliches Modell der gegenseitigen Aufklärung und Polizierung, das im Begriff des guten „Umgangs“ zusammengefasst werden kann - nicht nur für Lais „das einzige Mittel wie sie selbst entwickelt und gebildet werden kann“. ${ }^{56}$ Denn die griechischen Frauen waren natürlich genauso in ihren Haushalt eingesperrt wie ihre orientalischen Genossinnen im Harem; sie hatten weder über die Familien hinausgehende männliche Kontakte noch gar eine Möglichkeit zur Teilhabe am öffentlichen oder politischen Leben. Dagegen verstößt bereits Lais mit ihrem halb-öffentlichen Salon; aber auch für Aristipp ist es selbstverständlich, seinen Familienkreis für Gäste und Besucher aus der Fremde zu öffnen - und das nicht nur im Interesse der Frauen, sondern auch dem geselliger Kultur und wechselseitiger Zivilisierung:

Ohne sie würden wir nur mit halbem Mute fröhlich sein können; denn sie sind uns so unentbehrlich als Pindars Grazien den Göttern. ${ }^{57}$

Dieses Konzept des „Umgangs“, so lebensweltlich und trivial es erscheinen mag, weist auf einen wichtigen poetologischen Aspekt eines polyperspektivischen Briefromans zurück: Er erzeugt durch die Ausrichtung auf den Leser - den einzigen, der alle Briefe lesen kann! - eine dem gesellschaftlichen Umgang durchaus ähnliche Situation: Verschiedene Figuren werden ihm sympathischer sein als andere, verschiedene Positionen werden ihm näher liegen als andere, aber gleichwohl ist er auch mit weniger sympathischen Figuren und ihm eher fremden Ansichten konfrontiert, ohne dass ein Autor oder auch nur ein Herausgeber für ihn kommentiert, bewertet oder entscheidet. Er wird also ein Lektüreverhalten entwickeln müssen, das sich wesentlich von dem identifikatorischen (beispielsweise eines empfindsamen Briefromans) unterscheidet und das Aspekte der kognitiven Distanz mit solchen von emotionalem Engagement verbindet.

54. Vgl. Heinz, Narrative Kulturkonzepte, Kap. 5.3.1.

55. AZ, S. 102.

56. AZ, S. 103.

57. AZ, S. 592. 


\section{Vom ,Geist' des polyperspektivischen Briefromans - zur Zusammenfassung}

Montesquieus Persische Briefe und Wielands Aristipp-Roman demonstrieren in verschiedenen Varianten, wie Zivilisierung und polyperspektivischer Briefroman zusammenhängen. Beide Autoren teilen dabei, zum ersten, die Abneigung gegen allzu weitgehende philosophische Verallgemeinerungen und betonen demgegenüber den Wert des historisch Unterschiedlichen, des Besonderen von Völkern wie Personen; das ist die Basis ihrer jeweiligen Geschichtsphilosophie, wie sie sich bei Wieland in seinen diversen kulturtheoretischen Essays ebenso äußert wie in Montesquieus späterem Hauptwerk, dem Esprit des Loix. Gleichwohl sehen sie bei aller konkreten kulturellen Vielfalt auch eine gemeinsame Basis in einem fiktiven Urzustand der Menschheit und den damit verbundenen, substantiell guten natürlichen Gefühlen, wie sie in der Troglodyten-Geschichte exemplarisch dargestellt sind. Der polyperspektivische Briefroman kommt dieser Haltung dadurch entgegen, dass ihn ihm unterschiedliche Briefschreiber mit unterschiedlichen Überzeugungen miteinander in Kontakt treten, ohne dass eine bestimmte Position von vornherein als einzig wahre erscheint; der Leser muss selbst urteilen, und zwar auf der natürlichen Basis seiner Empathie mit allen Menschen sowie mit Hilfe seiner eigenen geselligen Bildung.

Montesquieu und Wieland teilen, zum zweiten, die Überzeugung, dass abstrakte philosophische Erkenntnisse fühlbar gemacht werden müssen, um eine aufklärerisch-pädagogische Wirkung beim Leser zu erzielen - was für beide die unbestrittene Hauptfunktion von Literatur und Philosophie ist. Durch eine allzu starke Emotionalisierung (wie sie wenig später der monoperspektivische, empfindsame Briefroman vorführen wird) entsteht die Gefahr, dass über der Identifikation die reflexive Distanz verloren geht, die für eine pädagogische Wirkung im Sinne der Aufklärung - nämlich die Einübung des Selbstdenkens - unabdingbar ist. Durch eine allzu starke Theoretisierung hingegen (wie in der philosophischen Abhandlung) geht das emotionale Antriebspotential verloren, das theoretische Erkenntnisse erst praktisch werden lässt. Der polyperspektivische Briefroman kommt dieser Intention dadurch entgegen, dass er sowohl digressivreflexive Passagen wie emotional-narrative enthält und diese sich gegenseitig beleuchten.

Montesquieu und Wieland teilen, zum dritten, die Überzeugung, dass die Zivilisierung des Einzelnen wie der Gesellschaft nur auf der Basis eines weitgehend herrschaftsfreien Verhältnisses der Menschen möglich ist; das bedeutet aber keine grenzenlose Freiheit oder individuelle Willkür, sondern eine freiwillig begrenzte und verantwortete Freiheit, die in der Mäßigung zugunsten der Interessen anderer ihr natürliches Gegengewicht findet. Der (von Natur aus unmäßige) Despotismus demonstriert, dass Gewaltherrschaft immer in Unfreiheit mündet, und zwar auch für die Herrschenden selbst. Während die Beherrschten sich wenigstens theoretisch ihre innere Freiheit erhalten können, wird der Despot zum Sklave seiner eigenen menschenfeindlichen Handlungen, die ihn zwingen, seine von 
Natur aus menschenfreundlichen Gefühle nicht nur zu unterdrücken, sondern letztlich zu vergewaltigen: Despotismus ist gelernte Empathie-Unfähigkeit. Der polyperspektivische Briefroman führt dem Leser demgegenüber nicht nur seine eigene moralische Freiheit vor Augen; er ermöglicht ihm auch eine Einübung in die Praxis des Rollentauschs (der Geschlechter ebenso wie der Kulturen oder Religionen), er gibt ihm die Möglichkeit, die Welt in all ihrer Vielfalt durch die Augen Anderer zu sehen - was nicht nur zu neuen Erkenntnissen, sondern auch zu stärkerer Empathiefähigkeit führt.

Empathie jedoch, die nicht nur mit dem Kopf erkannte, sondern mit dem Herzen gefühlte Gemeinsamkeit mit allen anderen Mitgliedern einer politischen Gemeinschaft oder gar der ganzen Menschheit, ist für beide Autoren die Basis jeder gelingenden politischen Organisationsform, welche äußerliche Form sie auch haben mag. Es ist, mit Montesquieu gesprochen, der „Geist der Gesetze“, der entscheidend für einen Staat ist, nicht ihr Buchstabe. Schon am 18. Mai 1764 hatte Wieland an seinen Freund Johann Georg Zimmermann geschrieben: „Es wird in unsern Tagen überall der Gebrauch Bücher in Form des Esprit des Loix zu schreiben, so wie es vor zwanzig Jahren Mode war, selbst die Theorie der PastetenBeckerey in geometrische Lehrart zu bringen. Schade, daß Montesquieu's Geist nicht eben so gut nachgeäfft werden kann als seine Methode“. ${ }^{58}$ Der Aristipp ist zweifellos ein polyperspektivischer Briefroman in dem zivilisatorischen und mäßigenden Geist, den die Persischen Briefe gestiftet haben - und der bis heute, aller Bildung und Zivilisierung zum Trotz, leider allzu wenig Nachfolger gefunden hat.

58. Wielands Briefwechsel. Bd. 3: Briefe der Biberacher Amtsjahre, hrsg. von Renate Petermann, Berlin, Akademie, 1975, S. 268. Wielands frühe Montesquieu-Lektüre ist übrigens auch anderweitig mehrfach bezeugt; so sah er in seinem frühen pädagogischen Konzept Plan einer Academie, zu Bildung des Verstandes und des Herzens junger Leute (1758) vor, in der dortigen dritten Klasse solle der „Esprit des Loix erklärt werden, als ein Werk, welches die wahren Schlüssel zu allen Gesezen, die gründlichste Politik, und beynahe alles enthält, was von der Staatsverfassung, den Gesezen, Sitten und der Religion aller Völker in allen Zeiten zu wissen der Mühe werth ist“ (Wielands Werke, Abt. 1, Bd. 4: Prosaische Jugendwerke, hrsg. von Fritz Homeyer und Hugo Bieber, Berlin, Weidmann, 1916, S. 196). Vgl. auch Vierhaus, Montesquieu in Deutschland, S. 12 und 18, zum Übersetzungsproblem und zur Wirkungsgeschichte des Begriffs ,Geist' in der deutschen politischen Diskussion. 